\title{
NEW BOUNDS FOR SPHERICAL TWO-DISTANCE SETS
}

\author{
ALEXANDER BARG* AND WEI-HSUAN YU ${ }^{\dagger}$
}

\begin{abstract}
A spherical two-distance set is a finite collection of unit vectors in $\mathbb{R}^{n}$ such that the distances between any two distinct vectors assume only two values. We use the semidefinite programming method to compute improved estimates of the maximum size of spherical two-distance sets. Exact answers are found for dimensions $n=23$ and $40 \leq n \leq 93(n \neq$ $46,78)$ where previous results gave divergent bounds.
\end{abstract}

\section{INTRODUCTION}

This paper is devoted to the application of the semidefinite programming method to estimates of the size of the largest possible two-distance set on the sphere $S^{n-1}(\mathbb{R})$. A spherical two-distance set is a finite collection $\mathcal{C}$ of unit vectors in $\mathbb{R}^{n}$ such that the set of distances between any two distinct vectors in $\mathcal{C}$ has cardinality two. Estimating the maximum size $g(n)$ of such a set is a classical problem in distance geometry that has been studied for several decades.

We begin with an overview of known results. A lower bound on $g(n)$ is obtained as follows. Let $e_{1}, \ldots, e_{n+1}$ be the standard basis in $\mathbb{R}^{n+1}$. The points $e_{i}+e_{j}, i \neq j$ form a spherical two-distance set in the plane $x_{1}+\cdots+x_{n+1}=2$ (after scaling), and therefore

$$
g(n) \geq n(n+1) / 2, \quad n \geq 2 .
$$

The first major result for upper bounds was obtained by Delsarte, Goethals, and Seidel [7]. They proved that, irrespective of the actual values of the distances, the following "harmonic" bound holds true:

$$
g(n) \leq n(n+3) / 2 .
$$

They also showed that this bound is tight for dimensions $n=2,6,22$ where it is attained by sets of equiangular lines. Moreover, the results of [7], Bannai et al. [4], and Nebe and Venkov [16] imply that $g(n)$ can attain the harmonic bound only if $n=(2 m+1)^{2}-3, m \geq 1$ with the exception of an infinite sequence of values of $m$ that begins with $m=3,4,6,10,12,22,38,30,34,42,46$. Therefore, unless $n$ is of the above form, $g(n) \leq n(n+3) / 2-1$. These results are proved using the link between 2-distance sets and tight spherical 4-designs established in [7].

Another advance in estimating the function $g(n)$ was made by Musin [15]. Let $\mathcal{C}=\left\{z_{1}, z_{2}, \ldots\right\}$ and suppose that $z_{i} \cdot z_{j} \in\{a, b\}, i \neq j$, where $2-2 a, 2-2 b$ are the values of the squared distances between the points. Musin proved that

$$
|\mathcal{C}| \leq n(n+1) / 2 \quad \text { if } a+b \geq 0 .
$$

He then used Delsarte's linear programming method to prove that $g(n)=n(n+1) / 2$ if $7 \leq n \leq 39, n \neq 22,23$.

Here we make another step for spherical two-distance sets, extending the range of dimensions in which the bound (1) is tight. The state of the art for $g(n)$ can be summarized as follows.

Theorem 1.1. We have $g(2)=5, g(3)=6, g(4)=10, g(5)=16, g(6)=27, g(22)=275$,

$$
\begin{aligned}
n(n+1) / 2 \leq g(n) & \leq n(n+3) / 2-1, \quad n=46,78 \\
g(n) & =n(n+1) / 2, \quad 7 \leq n \leq 93, n \neq 22,46,78,
\end{aligned}
$$

and $4465 \leq g(94) \leq 4492$. If $n \geq 95$, then $g(n) \leq n(n+3) / 2$ or $n(n+3) / 2-1$ as detailed in the remarks after Eq. (2) above.

Date: December 29, 2012. Research supported in part by NSF grants DMS1101697, CCF0916919, and CCF0830699 and NSA grant H98230101013.

* Dept. of Electrical and Computer Engineering and Institute for Systems Research, University of Maryland, College Park, MD 20742, and Institute for Information Transmission Problems, Russian Academy of Sciences, Moscow, Russia. Email: abarg@umd.edu.

$\dagger$ Dept. of Mathematics and Institute for Systems Research, University of Maryland, College Park, MD 20742. Email: mathyu @math.umd.edu. 
The part of this theorem that is established in the present paper relates to dimensions $n=23$ and $40 \leq n \leq$ $94, n \neq 46,78$. Our results are computational in nature and are obtained using the semidefinite programming method. The other parts of this theorem follow from the results in [7, 4, 15, 16].

As far as actual constructions of spherical two-distance sets are concerned, rather little is known beyond the set of midpoints of the edges of a regular simplex mentioned above. Another way of constructing such sets is to start with a set of equiangular lines in $\mathbb{R}^{n}$ [11]. If the angle between each pair of lines is $\alpha$, then taking one point from each pair of points on $S^{n-1}$ defined by the line, we obtain a two-distance set with $a=\alpha, b=-\alpha$. The largest possible number of equiangular lines in $\mathbb{R}^{n}$ is $n(n+1) / 2$ (this result is due to Gerzon, see [11]). This bound is attained for $n=3,7,23$. For instance, for $n=3$ the set of 6 lines is obtained from 6 diagonals of the icosahedron, which gives many ways of constructing inequivalent spherical two-distance sets of cardinality 6 . The only three instances in which the known spherical two-distance sets are of cardinality greater than $n(n+1) / 2$ occur in dimensions $n=2,6$ and 22 .

\section{Positive Definite MATRICES AND SDP BOUNDS}

A semidefinite program is an optimization problem of the form

$$
\max \left\{\langle X, C\rangle \mid X \succeq 0,\left\langle X, A_{i}\right\rangle=b_{i}, i=1, \ldots, m\right\},
$$

where $X$ is an $n \times n$ variable matrix, $A_{1}, \ldots, A_{m}$ and $C$ are given Hermitian matrices, $\left(b_{1}, \ldots, b_{m}\right)$ is a given vector and $\langle X, Y\rangle=\operatorname{trace}\left(Y^{*} X\right)$ is the inner product of two matrices. Semidefinite programming is an extension of linear programming that has found a range of applications in combinatorial optimization, control theory, distance geometry, and coding theory. General introduction to semidefinite programming is given, for instance, in [5].

The main problem addressed by the SDP method in distance geometry is related to deriving bounds on the cardinality of point sets in a metric space $\mathcal{X}$ with a given set of properties such as a given minimum separation between distinct points in the set. The SDP method has its roots in harmonic analysis of the isometry group of the metric space in question. It is broadly applicable in both finite and compact infinite spaces. Examples of the former include the Hamming and Johnson spaces, their $q$-analogs, other metric spaces on the set of $n$-strings over a finite alphabet, as well as the finite projective space. The main example in the infinite case is given by real and complex spheres, although the SDP method is also applicable in other compact homogeneous spaces. Working out the details in each example is a nontrivial task that includes analysis of irreducible modules in the space of functions $f: \mathcal{X} \rightarrow \mathbb{C}$ under the action of the isometry group $G$ of $\mathcal{X}$. The zonal matrices that arise in this analysis initially have large size that can be reduced relying on symmetries arising from the group action. This gives rise to an SDP optimization problem that is solved by computer for a given set of dimensions (the numerical part is also not straightforward and rather time-consuming). Foundations and analysis of particular cases have been the subject of a considerable number of research and overview publications in the last decade; see in particular recent surveys [2, 1] and references therein.

The origins of the SDP method and the discussed applications can be traced back to the work of Delsarte [6] which introduced the machinery of association schemes in the analysis of point configurations (codes) in finite spaces. Delsarte derived linear programming (LP) bounds on the cardinality of a set of points in the space under the condition on the minimum separation of distinct points in the set. Delsarte's results were linked to harmonic analysis and group representations in the works of Delsarte, Goethals and Seidel [7] (for the case $S^{n-1}$ ) and Kabatyansky and Levenshtein [9] (for general compact symmetric spaces).

From now on we focus on the case $\mathcal{X}=S^{n-1}$. Let $G_{k}^{(n)}(t), k=0,1, \ldots$ denote the Gegenbauer polynomials of degree $k$. They are defined recursively as follows: $G_{0}^{(n)} \equiv 1, G_{1}^{(n)}(t)=t$, and

$$
G_{k}^{(n)}(t)=\frac{(2 k+n-4) t G_{k-1}^{(n)}(t)-(k-1) G_{k-2}^{(n)}(t)}{k+n-3}, \quad k \geq 2 .
$$

Delsarte et al. [7] showed that for any finite set of points $\mathcal{C} \subset S^{n-1}$

$$
\sum_{(x, y) \in \mathcal{C}^{2}} G_{k}^{(n)}(x \cdot y) \geq 0, \quad k=1,2, \ldots
$$

The proof of this inequality in [7] used the addition formula for spherical harmonics. An earlier, geometric proof of (7) had been given by Schoenberg [19], although his work was not known to researchers in the area discussed until at least the 1990s.

Positivity conditions (7) give rise to the LP bound on the cardinality of spherical two-distance sets. 
Theorem 2.1. (Delsarte et al. [7]) Let $\mathcal{C} \subset S^{n-1}$ be a finite set and suppose that $x \cdot y \in\{a, b\}$ for any $x, y \in \mathcal{C}$. Then

$$
|\mathcal{C}| \leq \max \left\{1+\alpha_{1}+\alpha_{2}: 1+\alpha_{1} G_{i}^{(n)}(a)+\alpha_{2} G_{i}^{(n)}(b) \geq 0, i=0,1, \ldots, p ; \alpha_{j} \geq 0, j=1,2\right\} .
$$

In this theorem $\alpha_{1}, \alpha_{2}$ are the optimization variables that refer to the number of ordered pairs of points in $\mathcal{C}$ with inner product $a$ and $b$, respectively. For instance, $\alpha_{1}=|\mathcal{C}|^{-1} \sharp\left\{\left(z_{1}, z_{2}\right) \in \mathcal{C}^{2}: z_{1} \cdot z_{2}=a\right\}$, This theorem is a specialization of a more general LP bound on spherical codes of [7, 9].

Applications of semidefinite programming in coding theory and distance geometry gained momentum after the pioneering work of Schrijver [20] that derived SDP bounds on codes in the Hamming and Johnson spaces. Schijver's approach was based on the so-called Terwilliger algebra of the association scheme and formed a far-reaching generalization of the work of Delsarte [6]. Elements of the groundwork for SDP bounds in the Hamming space were laid by Dunkl [8], although this connection was also made somewhat later [21]. We refer to [13] for a detailed general survey of the approach via association schemes and further references.

SDP bounds for the real sphere were derived by Bachoc and Vallentin [3] in the context of the kissing number problem. The kissing number $k(n)$ is the maximum number of unit spheres that can touch a unit sphere without overlapping, i.e. the maximum number of points on the sphere such that the angular separation between any pair of them is at least $\pi / 3$. Following [3], define a $(p-k+1) \times(p-k+1)$ matrix $Y_{k}^{n}(u, v, t), k \geq 0$ by setting

$$
\left(Y_{k}^{n}(u, v, t)\right)_{i j}=u^{i} v^{j}\left(\left(1-u^{2}\right)\left(1-v^{2}\right)\right)^{k / 2} G_{k}^{(n-1)}\left(\frac{t-u v}{\sqrt{\left(1-u^{2}\right)\left(1-v^{2}\right)}}\right)
$$

where $p$ is a positive integer, and a matrix $S_{k}^{n}(u, v, t)$ by setting

$$
S_{k}^{n}(u, v, t)=\frac{1}{6} \sum_{\sigma} Y_{k}^{n}(\sigma(u, v, t))
$$

where the sum is over all permutations on 3 elements. Note that $\left(S_{k}^{n}(1,1,1)\right)_{i j}=0$ for all $i, j$ and all $k \geq 1$. One of the main results of [3] is that for any finite set of points $\mathcal{C} \subset S^{n-1}$

$$
\sum_{(x, y, z) \in \mathcal{C}^{3}} S_{k}^{n}(x \cdot y, x \cdot z, y \cdot z) \succeq 0
$$

The matrices $S_{k}^{n}$ play the role of the constraints $A_{i}$ in the general SDP problem (6). Positivity constraints (8) give rise to a general SDP bound on the cardinality of point sets obtained in [3], where it was used to improve upper bounds on $k(n)$ in small dimensions. In the next section we state a specialization of this bound for the case of 2-distance sets.

As a final remark, we note that constraints (7) arise from the unrestricted action of $G$ on $S^{n-1}$. Constraints (8) are obtained by considering only actions that fix an arbitrary given point on the sphere. Further SDP bounds can be obtained by considering zonal matrices that arise from actions that fix any given number of points; however even for two points, actual evaluation of the bounds requires significant computational effort [14].

\section{THE BOUNDS}

The general SDP bound on spherical codes of [3] specializes to our case as follows.

Theorem 3.1. Let $\mathcal{C}$ be a spherical two-distance set with inner products a and $b$. Let $p$ be a positive integer. The cardinality $|\mathcal{C}|$ is bounded above by the solution of the following semidefinite programming problem:

$$
\begin{gathered}
1+1 / 3 \max \left(x_{1}+x_{2}\right) \\
\text { subject to } \\
\left(\begin{array}{ll}
1 & 0 \\
0 & 0
\end{array}\right)+\frac{1}{3}\left(\begin{array}{ll}
0 & 1 \\
1 & 1
\end{array}\right)\left(x_{1}+x_{2}\right)+\left(\begin{array}{ll}
0 & 0 \\
0 & 1
\end{array}\right)\left(x_{3}+x_{4}+x_{5}+x_{6}\right) \succeq 0 \\
3+G_{i}^{(n)}(a) x_{1}+G_{i}^{(n)}(b) x_{2} \geq 0, \quad i=1,2, \ldots, p \\
S_{i}^{n}(1,1,1)+S_{i}^{n}(a, a, 1) x_{1}+S_{i}^{n}(b, b, 1) x_{2}+S_{i}^{n}(a, a, a) x_{3} \\
+S_{i}^{n}(a, a, b) x_{4}+S_{i}^{n}(a, b, b) x_{5}+S_{i}^{n}(b, b, b) x_{6} \succeq 0, \quad i=0,1, \ldots, p \\
x_{j} \geq 0, j=1, \ldots, 6,
\end{gathered}
$$

where $S_{i}(\cdot, \cdot, \cdot)$ are $(p-i+1) \times(p-i+1)$ matrices defined in (8). 
In this theorem the variables $x_{1}, x_{2}$ refer to the number of ordered pairs of vectors in $\mathcal{C}$ with inner product $a$ and $b$ respectively; namely we have $x_{i}=3 \alpha_{i}, i=1,2$. We note that the SDP problem seeks to optimize the same linear form as the LP problem, but adds more constraints on the configuration. Because of this, Theorem 3.1 usually gives tighter bounds than Theorem 2.1. This fact is evident from the table below and is also known from the calculation of kissing numbers in [3].

3-A. Calculation of the bound. Several remarks are in order. First, implementation of SDP for two-distance sets differs from earlier computations in [3, 14] in that in our case there are no limits on the minimum separation of the points. Next, we restrict our calculations to the case $p \leq 5$ as no improvement is observed for larger values. Finally, by a result Larman et al. [10], if $|\mathcal{C}| \geq 2 n+3$ then the inner products $a, b$ are related by $b=b_{k}(a)=(k a-1) /(k-1)$ where $k \in\{2, \ldots,\lfloor(1+\sqrt{2 n}) / 2\rfloor\}$ is an integer. Thus we obtain a family of SDP bounds parametrized by $a$. Since $b_{k}(a) \geq-1, a+b_{k}(a)<0$, we get that $a \in I_{k}:=\left[0, \frac{1}{2 k-1}\right)$. Moreover, if $-1 \leq b<a \leq 0$, then $|\mathcal{C}|$ cannot be large by the Rankin bounds [18], and if $a+b \geq 0$ then $|\mathcal{C}|$ is bounded by (3). We conclude as follows.

Theorem 3.2. Let $\operatorname{SDP}(a)$ be the solution of the $\operatorname{SDP}$ problem (10)-(13), where $b=b_{k}(a)$. Let $\mathcal{C}$ be a spherical two-distance set with inner products $a, b$, then

$$
|\mathcal{C}| \leq \begin{cases}n(n+1) / 2, & a+b \geq 0 \\ \operatorname{SDP}(a), & a \in I_{k} \\ n+1, & -1 \leq b<a<0 .\end{cases}
$$

For instance, for $n=23, k=3$ we obtain that $I_{k}=[0,0.2)$. Partitioning $I_{k}$ into a number of small segments, we plot the value $\operatorname{SDP}(a)$ as a function of $a$ evaluated at the nodes of the partition. The result is shown in Fig. 1(a) A part of the segment around the maximum appears in Fig. 1(b). This computation gives an indication of the answer, but in principle the value $\operatorname{SDP}(a)$ could oscillate between the nodes of the partition. Ruling this out requires perturbation analysis of the SDP problem which is not immediate.

3-A.1. Dual problem. The dual problem of (10)-(13) has the following form.

$$
\begin{gathered}
1+\min \left\{\sum_{i=1}^{p} \alpha_{i}+\beta_{11}+\left\langle F_{0}, S_{0}^{n}(1,1,1)\right\rangle\right\} \\
\text { subject to } \\
\left(\begin{array}{cc}
\beta_{11} & \beta_{22} \\
\beta_{12} & \beta_{22}
\end{array}\right) \succeq 0 \\
2 \beta_{12}+\beta_{22}+\sum_{i=1}^{p}\left(\alpha_{i} G_{i}^{(n)}(a)+3\left\langle F_{i}, S_{i}^{n}(a, a, 1)\right\rangle\right) \leq-1 \\
2 \beta_{12}+\beta_{22}+\sum_{i=1}^{p}\left(\alpha_{i} G_{i}^{(n)}(b)+3\left\langle F_{i}, S_{i}^{n}(b, b, 1)\right\rangle\right) \leq-1 \\
\beta_{22}+\sum_{i=0}^{p}\left\langle F_{i}, S_{i}^{n}\left(y_{1}, y_{2}, y_{3}\right)\right\rangle \leq 0 \\
\text { where }\left(y_{1}, y_{2}, y_{3}\right) \in\{(a, a, a),(a, a, b),(a, b, b),(b, b, b)\} \\
\alpha_{i} \geq 0, F_{i} \succeq 0, i=1, \ldots, p .
\end{gathered}
$$

We need to estimate from above the maximum value of this problem over $a \in I_{k}=\left[a_{1}, a_{2}\right]$. Accounting for a continuous value set of the parameter in SDP problems is a challenging task. We approach it by employing the sumof-squares method. Constraints (15)-(17) impose positivity conditions on some univariate polynomials of $a$ for $a \in I_{k}$. The following sequence of steps transforms the constraints to semidefinite conditions. Observe that a polynomial $f(a)$ of degree at most $m$ satisfies $f(a) \geq 0$ for $a \in I_{k}$ if and only if the polynomial of degree at most $2 m$

$$
f^{+}(a)=\left(1+a^{2}\right)^{m} f\left(\frac{a_{1}+a_{2} a^{2}}{1+a^{2}}\right) \geq 0
$$


for all $a \in \mathbb{R}$. Next, a polynomial nonnegative on the entire real axis can be written as a sum of squares, $f(x)=$ $\sum_{i} r_{i}^{2}(x)$, where the $r_{i}$ are polynomials. Further, by a result of Nesterov [17], a polynomial $f(x)$ of degree $2 m$ is a sum of squares if and only if there exists a positive semidefinite matrix $Q$ such that $f=X Q X^{t}$, where $X=$ $\left(1, x, x^{2}, \ldots, x^{m}\right)$. Thus, constraints (15)-(17) can be transformed to semidefinite conditions.

As a result, we obtain an SDP problem that can be solved by computer. We solved the resulting problem for $7 \leq n \leq 96$ using the Matlab toolbox SOSTOOLS [22] in the YALMIP environment [12]. An advantage in using SOSTOOLS is that it accepts $a$ as an SDP variable, thereby accounting for all the values of $a$ in the segment. Thus, we obtain the value $\max \operatorname{SDP}(a), a \in I_{k}$. However, this may impose excessive constraints on the value of the SDP problem because all the conditions for different values of $a$ are involved at the same time. To work around this accumulation, we use a sub-partitioning of the segment $I_{k}$ into smaller segments. For each of them, SOSTOOLS outputs the largest value of the minimum of the SDP problem over all $a$ in the segment. It turns out that, in many cases, the maximum of these solutions is smaller than $\max \operatorname{SDP}(a), a \in I_{k}$ computed directly by the package. The estimates of the answer computed from the primal problem serve as a guidance of the needed step length of the partition. The solution of the sum-of-squares SDP optimization problem provides a rigorous proof for the estimates obtained by discretizing the primal problem (10)-(13). For instance, for $n=23$ we partition $I_{3}$ into 20 subsegments, finding 276.5 as the maximum value of the dual SDP problem for $a \in I_{3}$, etc.

3-A.2. Results. The results of the calculation are summarized in the table below. The part of the table for $7 \leq n \leq 40$, except for the values of the SDP bound, is from [15]. The improvement provided by Theorem 3.1 over the LP bound is quite substantial even for relatively small dimensions. The LP bound is above $n(n+1) / 2$ for $n \geq 40$ and is not included starting with $n=41$. The cases $n=46,78$ and $n \geq 94$ are not resolved by SDP, although for $n=94$ we still obtain an improvement over the harmonic bound (2). The value of $k$ shown in the table accounts for the largest value of the SDP problem among the possible choices of $k$. This guarantees that the value $\operatorname{SDP}(a)$ is equal to or smaller than the number in the table for all the possible values of the inner products $a, b$ in the point set.

Notice that for $n=46,78$ the SDP bound coincides with the bound (2). For $n=23$ the results of [15] leave two possibilities, $g(n)=276$ and 277. The SDP bound resolves this for the former, establishing the corresponding part of the claim in (5). As is seen from Fig. 1(b), the largest value of $\operatorname{SDP}(a)$ is attained for $a=0.2$ and is equal to 276. This case corresponds to 276 equiangular lines in $R^{23}$ with angle $\arccos 0.2$, which can be constructed either using strongly regular graphs or the Leech lattice (see [11] for details).

Acknowledgment: We are grateful to Chao-Wei Chen and Johan Löfberg for their significant help with the Matlab implementation.

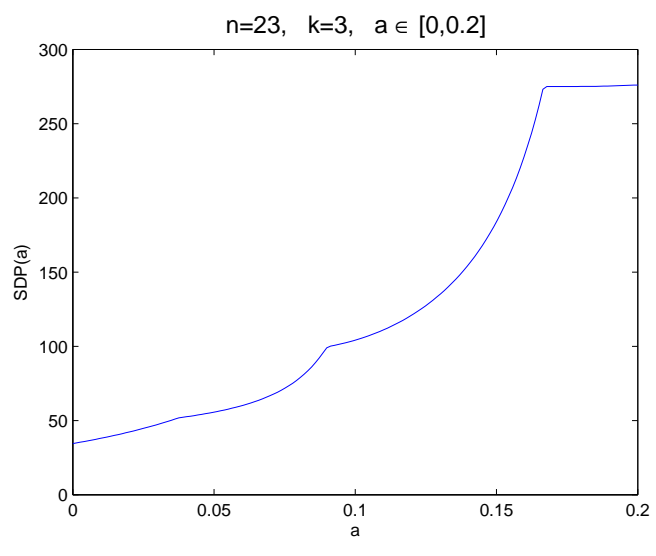

(a) The value $\operatorname{SDP}(a)$ for $n=23$

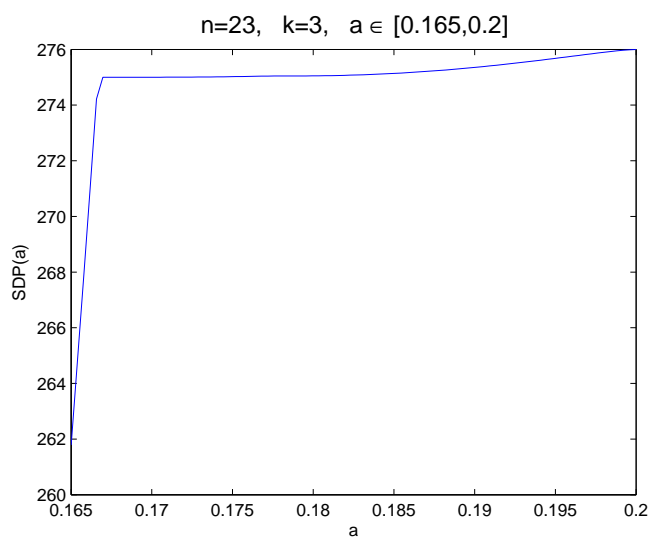

(b) The neighborhood of the maximum

FIGURE 1. Evaluation of the SDP bound on $g(23)$

\section{REFERENCES}

[1] C. Bachoc, Semidefinite programming, harmonic analysis and coding theory, arXiv:0909.4767 2009, 43pp.

[2] C. Bachoc, D.C. Gijswijt, A. Schrijver, and F. Vallentin, Invariant semidefinite programs, Handbook on semidefinite, conic and polynomial optimization, Internat. Ser. Oper. Res. Management Sci., vol. 166, Springer, New York, NY, 2012, pp. 219-269. 
A. BARG AND W.-H. YU

[3] C. Bachoc and F. Vallentin, New upper bounds for kissing numbers from semidefinite programming, J. Amer. Math. Soc. 21 (2008), 909-924.

[4] E. Bannai, A. Munemasa, and B. Venkov, The nonexistence of certain tight spherical designs, St. Petersburg Math. J. 16 (2005), $609-625$.

[5] A. Ben-Tal and A. Nemirovski, Lectures on modern convex optimization. Analysis, algorithms, and engineering applications, SIAM, Philadelphia, PA, 2001.

[6] P. Delsarte, An algebraic approach to the association schemes of coding theory, Philips Research Repts Suppl. 10 (1973), 1-97.

[7] P. Delsarte, J. M. Goethals, and J. J. Seidel, Spherical codes and designs, Geometriae Dedicata 6 (1977), 363-388.

[8] C. Dunkl, A Krawtchouk polynomial addition theorem and wreath products of symmetric groups, Indiana Univ. Math. J. 25 (1976), no. 4, $335-358$.

[9] G.A. Kabatyansky and V.I. Levenshtein, Bounds for packings on the sphere and in the space, Problems of Information Transmission 14 (1978), no. $1,3-25$.

[10] D. G. Larman, C. A. Rogers, and J. J. Seidel, On two-distance sets in Euclidean space, Bull. London Math. Soc. 9 (1977), $261-267$.

[11] P. W. H. Lemmens and J. J. Seidel, Equiangular lines, Journal of Algebra 24 (1973), 494-512.

[12] J. Löfberg, http://users.isy.liu.se/johanl/yalmip/

[13] W. J. Martin and H. Tanaka, Commutative association schemes, European J. Combin. 30 (2009), no. 6, 1497-1525.

[14] H. D. Mittelman and F. Vallentin, High accuracy semidefinite programming bounds for kissing numbers, Experimental Mathematics 19 (2010), $174-178$.

[15] O. R. Musin, Spherical two-distance sets, J. Combin. Theory Ser. A 116 (2009), no. 4, 988-995.

[16] G. Nebe and B. Venkov, On tight spherical designs, arXiv:1201.1830, 2012, 8pp.

[17] Yu. Nesterov, Squared functional systems and optimization problems, High Performance Optimization (H. Frenk, K. Roos, T. Terlaky, and S. Zhanget, eds.), Kluwer, 2000, pp. 405-440.

[18] R. A. Rankin, The closest packing of spherical caps in n dimensions, Proc. Glasgow Math. Assoc. 2 (1955), 139-144.

[19] I.J. Schoenberg, Positive definite functions on spheres, Duke Math. J. 9 (1942), 96-107.

[20] A. Schrijver, New code upper bounds from the Terwilliger algebra and semidefinite programming, IEEE Trans. Inform. Theory 51 (2005), no. $8,2859-2866$.

[21] F. Vallentin, Symmetry in semidefinite programs, Linear Algebra Appl. 430 (2009), no. 1, 360-369.

[22] http://www.cds.caltech.edu/sostools/ 
Bounds on two-distance sets. The starred rows correspond to dimensions for which the value of $g(n)$ is not known exactly.

\begin{tabular}{|c|c|c|c|c|c|c|c|c|}
\hline$n$ & LP bound & SDP bound & $n(n+1) / 2$ & $k$ & $n$ & SDP bound & $n(n+1) / 2$ & $k$ \\
\hline 7 & 28 & 28 & 28 & 2 & 52 & 1128 & 1378 & 4 \\
\hline 8 & 31 & 28 & 36 & 2 & 53 & 1128 & 1431 & 4 \\
\hline 9 & 34 & 29 & 45 & 2 & 54 & 1128 & 1485 & 4 \\
\hline 10 & 37 & 29 & 55 & 2 & 55 & 1128 & 1540 & 4 \\
\hline 11 & 40 & 29 & 66 & 2 & 56 & 1128 & 1596 & 4 \\
\hline 12 & 44 & 28 & 78 & 2 & 57 & 1162 & 1653 & 2 \\
\hline 13 & 47 & 29 & 91 & 3 & 58 & 1200 & 1711 & 2 \\
\hline 14 & 52 & 35 & 105 & 2 & 59 & 1240 & 1770 & 2 \\
\hline 15 & 56 & 41 & 120 & 3 & 60 & 1282 & 1830 & 2 \\
\hline 16 & 61 & 50 & 136 & 3 & 61 & 1324 & 1891 & 2 \\
\hline 17 & 66 & 60 & 153 & 3 & 62 & 1372 & 1953 & 2 \\
\hline 18 & 76 & 75 & 171 & 3 & 63 & 1428 & 2016 & 2 \\
\hline 19 & 96 & 95 & 190 & 3 & 64 & 1482 & 2080 & 2 \\
\hline 20 & 126 & 124 & 210 & 3 & 65 & 1540 & 2145 & 2 \\
\hline 21 & 176 & 174 & 231 & 3 & 66 & 1604 & 2211 & 2 \\
\hline 22 & 275 & 275 & 253 & 3 & 67 & 1672 & 2278 & 2 \\
\hline 23 & 277 & 276 & 276 & 3 & 68 & 1745 & 2346 & 2 \\
\hline 24 & 280 & 276 & 300 & 3 & 69 & 1822 & 2415 & 2 \\
\hline 25 & 284 & 276 & 325 & 3 & 70 & 1907 & 2485 & 2 \\
\hline 26 & 288 & 276 & 351 & 3 & 71 & 1999 & 2556 & 2 \\
\hline 27 & 294 & 276 & 378 & 3 & 72 & 2097 & 2628 & 2 \\
\hline 28 & 299 & 276 & 406 & 3 & 73 & 2206 & 2701 & 2 \\
\hline 29 & 305 & 276 & 435 & 3 & 74 & 2325 & 2775 & 2 \\
\hline 30 & 312 & 276 & 465 & 3 & 75 & 2394 & 2850 & 2 \\
\hline 31 & 319 & 276 & 496 & 3 & 76 & 2468 & 2926 & 2 \\
\hline 32 & 327 & 276 & 528 & 3 & 77 & 2542 & 3003 & 2 \\
\hline 33 & 334 & 276 & 561 & 3 & $78^{*}$ & 3159 & 3081 & 2 \\
\hline 34 & 342 & 276 & 595 & 3 & 79 & 3160 & 3160 & 4 \\
\hline 35 & 360 & 276 & 630 & 2 & 80 & 3160 & 3240 & 4 \\
\hline 36 & 416 & 276 & 666 & 2 & 81 & 3160 & 3321 & 4 \\
\hline 37 & 488 & 276 & 703 & 2 & 82 & 3160 & 3403 & 4 \\
\hline 38 & 584 & 276 & 741 & 2 & 83 & 3160 & 3486 & 4 \\
\hline 39 & 721 & 292 & 780 & 2 & 84 & 3185 & 3570 & 4 \\
\hline 40 & 928 & 315 & 820 & 2 & 85 & 3294 & 3655 & 4 \\
\hline 41 & & 341 & 861 & 2 & 86 & 3408 & 3741 & 4 \\
\hline 42 & & 370 & 903 & 2 & 87 & 3522 & 3828 & 4 \\
\hline 43 & & 422 & 946 & 2 & 88 & 3645 & 3916 & 4 \\
\hline 44 & & 540 & 990 & 2 & 89 & 3749 & 4005 & 4 \\
\hline 45 & & 736 & 1035 & 2 & 90 & 3905 & 4095 & 4 \\
\hline $46^{*}$ & & 1127 & 1081 & 2 & 91 & 4038 & 4186 & 4 \\
\hline 47 & & 1128 & 1128 & 2 & 92 & 4171 & 4278 & 4 \\
\hline 48 & & 1128 & 1176 & 2 & 93 & 4335 & 4371 & 4 \\
\hline 49 & & 1128 & 1225 & 2 & $94^{*}$ & 4492 & 4465 & 4 \\
\hline 50 & & 1128 & 1275 & 4 & $95^{*}$ & 4668 & 4560 & 4 \\
\hline 51 & & 1128 & 1326 & 4 & $96^{*}$ & 4828 & 4656 & 4 \\
\hline
\end{tabular}

